\title{
Social Distance Augmented Qualitative Trajectory Calculus for Human-Robot Spatial Interaction
}

\author{
Christian Dondrup ${ }^{1}$ and Nicola Bellotto ${ }^{1}$ and Marc Hanheide ${ }^{1}$
}

\begin{abstract}
In this paper we propose to augment a wellestablished Qualitative Trajectory Calculus (QTC) by incorporating social distances into the model to facilitate a richer and more powerful representation of Human-Robot Spatial Interaction (HRSI). By combining two variants of QTC that implement different resolutions and switching between them based on distance thresholds we show that we are able to both reduce the complexity of the representation and at the same time enrich QTC with one of the core HRSI concepts: proxemics. Building on this novel integrated QTC model, we propose to represent the joint spatial behaviour of a human and a robot employing a probabilistic representation based on Hidden Markov Models. We show the appropriateness of our approach by encoding different HRSI behaviours observed in a human-robot interaction study and show how the models can be used to represent and classify these behaviours using social distance-augmented QTC.
\end{abstract}

\section{INTRODUCTION}

Human-Robot Spatial Interaction (HRSI) is the study of joint movement of robots and humans through space. It is concerned with the investigation of models of the ways humans and robots manage their motions in vicinity to each other. These encounters might, for example, be socalled pass-by situations where human and robot aim to pass through a corridor trying to circumvent each other given spatial constraints (see Fig. 1). In order to resolve these kind of situations and pass through the corridor the human and the robot need to be aware of their mutual goals and have to have a way of negotiating who goes first or who goes to which side. Our work aims to equip a mobile robot with understanding of such HRSI situations and enable it to act accordingly.

In early works on mobile robotics humans have merely been regarded as static obstacles [1] that have to be avoided. More recently, the dynamic aspects of "human obstacles" has been taken into account, e.g. [2]. Currently, a large body of research is dedicated to answer the fundamental questions of HRSI and is producing navigation approaches which plan to explicitly move on more "socially acceptable and legible paths" [3], [4], [5]. The term "legible" here refers to the communicative - or interactive - aspects of motions which previously has widely been ignored in robotics research. According to Ducourant et al. [6], who investigated human

The research leading to these results has received funding from the European Community's Seventh Framework Programme under grant agreement No. 600623, STRANDS.

${ }^{1}$ Christian Dondrup, Nicola Bellotto, and Marc Hanheide are with the School of Computer Science, University of Lincoln, Lincoln, United Kingdom \{cdondrup, nbellotto, mhanheide\} alincoln.ac.uk
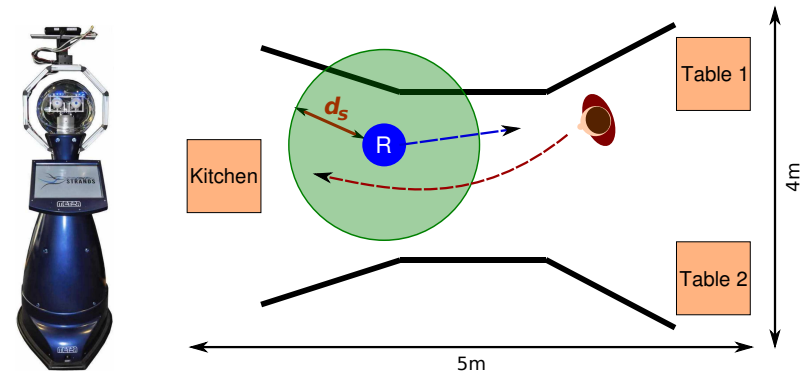

Fig. 1: Left: Robot. Hight: $1.72 \mathrm{~m}$, diameter: $\sim 61 \mathrm{~cm}$. Right: Head-on encounter. Robot ("R") tries to reach a table while the human (reddish figure) is trying to reach the kitchen. Experimental set-up: kitchen on the left and two tables on the right. Black lines represent the corridor. Circle around robot represents the distance threshold $d_{s}$.

spatial behaviour, humans also have to consider the actions of others as well when planning their own actions. Hence, moving around is also about communication and coordination of movements between two agents - at least when moving in close vicinity to one another, e.g. entering each others social or personal spaces [7].

For the analysis of HRSI, knowing the exact human and robot trajectories is often not necessary or even detrimental when trying to capture the "essence" of the interaction. Instead, it is more important to represent qualitatively how the agents move with respect to each other, in order to understand underlying social rules and conventions. In our previous work, we proposed a qualitative framework based on the analysis of relative position and movement direction between two interacting agents on a 2D environment [8], [9], [10]. In particular, to reduce the space domain and focus only on those terms relevant to HRSI, we adopted the well-defined set of symbols and relations provided by the Qualitative Trajectory Calculus (QTC), a formalism representing the relative motion of two points in space in a qualitative framework [11].

Social distances are an essential factor in HRSI as shown in Hall's proxemics theory [7] and numerous works on HRSI itself, e.g. [12]. So far these distances have not been represented in QTC which deprived it of the ability to generate appropriate behaviour regarding HRSI standards. Lichtenthäler et al. [13], for example, suggested to model distances explicitly by expanding the QTC representation to incorporate this and other quantitative measures. To preserve the qualitative nature and the resulting generalisability and simplicity of QTC representations we go beyond our pre- 


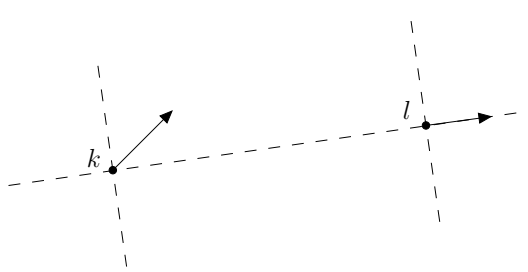

Fig. 2: Example of moving points $k$ and $l$. The respective $\mathrm{QTC}_{B}$ and $\mathrm{QTC}_{C}$ relations are $(-+)$ and $(-+-0)$.

vious work by proposing to model distance implicitly by transitioning between a coarse and a fine variant of QTC according to a distance threshold, e.g. Hall's personal space [7]. Instead of using "manually" crafted transitions between the different QTC variants [9] we propose a probabilistic model with increasing granularity of the QTC representation depending on the distance of human and robot trained from real world data. This does not only give the possibility to model this crucial HRSI metric in QTC but also makes use of more detailed action representation only when robot and human are in close vicinity to one another. Thereby, we also simplify our previously presented probabilistic model [10] by employing a rather coarse representation when the human and robot are far apart and only switch to finergrained representations when the two interactants are getting closer to one another. The main contribution of this work therefore is the enriching of QTC with distance measures, i.e. proxemics [7], while still preserving all the characteristics and properties of the underlying calculus. Combining a coarser and a finer variant of QTC to achieve this goal also creates a more compact representation of HRSI that is still complex enough to unambiguously represent the encounters observed in our user study.

\section{The Qualitative Trajectory Calculus}

\section{A. QTC Basic and QTC Double-Cross}

QTC belongs to the broad research area of qualitative spatial representation and reasoning [14], from which it inherits some of its properties and tools. There are several versions of QTC, depending on the number of factors considered (e.g. relative distance, speed, direction, etc.) and on the dimensions, or constraints, of the space where the points move. The simplest version, called QTC Basic $\left(\mathrm{QTC}_{B}\right)$, represents the relative motion of two points $k$ and $l$ with respect to the reference line connecting them (see Fig. 2). It uses a 2-tuple of qualitative relations $(a b)$, where each element can assume any of the values $\{-, 0,+\}$ as follows ${ }^{2}$ :

a) movement of $k$ with respect to $l$

- $: k$ is moving towards $l$

$0: k$ is stable with respect to $l$

$+: k$ is moving away from $l$

b) movement of $l$ with respect to $k$ : as above, but swapping $k$ and $l$

\footnotetext{
${ }^{2}$ The actual versions considered here are $\mathrm{QTC}_{B 11}$ and $\mathrm{QTC}_{C 21}$ [11], but for simplicity we refer to them as $\mathrm{QTC}_{B}$ and $\mathrm{QTC}_{C}$ respectively.
}

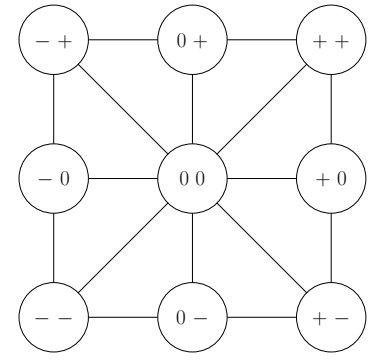

Fig. 3: CND of $\mathrm{QTC}_{B}$. Note that due to the original formulation [11], there are no direct transitions in the CND between some of the states that, at a first glance, appear to be adjacent (e.g. $(-0)$ and $(0-))$.

Therefore, the state set $S_{B}=\{(a, b): a, b \in\{-, 0,+\}\}$ for $\mathrm{QTC}_{B}$ has $\left|S_{B}\right|=3^{2}$ possible states and $\left|\tau_{B}\right|=$ $\left|\left\{s \rightsquigarrow s^{\prime}: s, s^{\prime} \in S_{B} \wedge s \neq s^{\prime}\right\}\right|=32$ legal transitions as defined in the Conceptual Neighbourhood Diagram ${ }^{3}$ (CND) shown in Fig. 3 [11]. By restricting the number of possible transitions - assuming continuous observations of both agents - a CND reduces the search space for subsequent states, and therefore the complexity of temporal QTC sequences.

Another version of the calculus, called QTC Double-Cross $\left(\mathrm{QTC}_{C}\right)$, extends the previous one to include also the side the two points move to, again with respect to the reference line connecting them (see Fig. 2). In addition to the 2-tuple $(a b)$ of $\mathrm{QTC}_{B}$, the relations $(c d)$ are considered, where each element can assume any of the values $\{-, 0,+\}$ as follows:

c) movement of $k$ with respect to $\overrightarrow{k l}$ $-: k$ is moving to the left side of $\overrightarrow{k l}$

$0: k$ is moving along $\overrightarrow{k l}$

$+: k$ is moving to the right side of $\overrightarrow{k l}$

d) movement of $l$ with respect to $\vec{l}$ : as above, but swapping $k$ and $l$

The resulting 4-tuple $(a b c d)$ representing the $\mathrm{QTC}_{C}$ state set $S_{C}=\{(a, b, c, d): a, b, c, d \in\{-, 0,+\}\}$, has $\left|S_{C}\right|=3^{4}$ states, and $\left|\tau_{C}\right|=\left|\left\{s \rightsquigarrow s^{\prime}: s, s^{\prime} \in S_{C} \wedge s \neq s^{\prime}\right\}\right|=1088$ legal transitions as defined in the corresponding CND [11].

As shown in [9], $\mathrm{QTC}_{B}$ and $\mathrm{QTC}_{C}$ can be "manually" combined to represent and reason about HRSIs. In the following section, however, we formalise and automatise this process.

\section{B. Integrating $Q T C_{B}$ and $Q T C_{C}$}

To achieve the desired implicit modelling of social distances and simplification of QTC state chains for HRSI we propose the integration of $\mathrm{QTC}_{B}$ and $\mathrm{QTC}_{C}$ referring to it as $\mathrm{QTC}_{B C}$.

The set of possible states for $\mathrm{QTC}_{B C}$ is a simple unification of the fused QTC variants. In the presented case the integrated $\mathrm{QTC}_{B C}$ states are defined as:

$$
S_{I}=S_{B} \cup S_{C}
$$

\footnotetext{
${ }^{3}$ We are adopting the notation $s_{1} \rightsquigarrow s_{2}$ for valid transitions according to the CND from [11].
} 
with $\left|S_{I}\right|=\left|S_{B}\right|+\left|S_{C}\right|=90$ states.

The transitions of $\mathrm{QTC}_{B C}$ include the unification of the transitions of $\mathrm{QTC}_{B}$ and $\mathrm{QTC}_{C}$ but also the transitions from $\mathrm{QTC}_{B}$ to $\mathrm{QTC}_{C}: \tau_{B C}=$ $\left\{s_{b} \rightsquigarrow s_{c}: s_{b} \in S_{B}, s_{c} \in S_{C}\right\}$ and from $\mathrm{QTC}_{C}$ to $\mathrm{QTC}_{B}$ : $\tau_{C B}=\left\{s_{c} \rightsquigarrow s_{b}: s_{b} \in S_{B}, s_{c} \in S_{C}\right\}$, respectively. This leads to the definition of the integrated QTC $_{B C}$ transitions as:

$$
\tau_{I}=\tau_{B} \cup \tau_{C} \cup\left(\tau_{B C} \cup \tau_{C B}\right)
$$

$\tau_{B C}$ and $\tau_{C B}$ are simply regarded as an increase or decrease in granularity. There are two different types of transitions:

1) Pseudo self-transitions where the values of $(a b)$ do not change, plus all possible combinations for the 2tuple $(c d):\left|S_{B}\right| \cdot 3^{2}=81$, e.g. $(++) \rightsquigarrow(++--)$ or $(++--) \rightsquigarrow(++)$.

2) Normal $\mathrm{QTC}_{B}$ transitions, plus all possible combinations for the 2-tuple $(c d):\left|\tau_{B}\right| \cdot 3^{2}=288$, e.g. $(+0) \rightsquigarrow(++--)$ or $(+0--) \rightsquigarrow(++)$.

Resulting into:

$$
\left|\tau_{B C}\right|+\left|\tau_{C B}\right|=2 \cdot(81+288)=738
$$

transitions between the two QTC variants. This leads to a total number of $\mathrm{QTC}_{B C}$ transitions of:

$$
\begin{aligned}
\tau_{I} & =\left|\tau_{B}\right|+\left|\tau_{C}\right|+\left(\left|\tau_{B C}\right|+\left|\tau_{C B}\right|\right) \\
& =32+1088+738 \\
& =1858
\end{aligned}
$$

These transitions depend on the previous and current euclidean distance of the two points $d(k, l)$ and the threshold $d_{s}$ representing an arbitrary social distance:

$$
\tau_{I}= \begin{cases}\tau_{B} & \text { if } d(k, l)_{t-1}>d_{s} \wedge d(k, l)_{t}>d_{s}, \\ \tau_{B C} & \text { else if } d(k, l)_{t-1}>d_{s} \wedge d(k, l)_{t} \leq d_{s}, \\ \tau_{C B} & \text { else if } d(k, l)_{t-1} \leq d_{s} \wedge d(k, l)_{t}>d_{s}, \\ \tau_{C} & \text { otherwise }\end{cases}
$$

These transitions, distances, and thresholds play a vital role in our probabilistic representation of HRSI which will be described in the following section.

\section{Probabilistic Model of $\mathrm{QTC}_{B C}$}

In previous work [10] we proposed a Hidden Markov Model (HMM) [15] based representation of QTC $_{C}$. This enabled us to represent actual sensor data by allowing for uncertainty in the recognition process. With this approach we were able to reliably classify head-on (see Fig. 1) and overtake ${ }^{4}$ scenarios and showed that the $\mathrm{QTC}_{C}$ representations of these two scenarios are significantly different from each other due to the distinctly different directions of travel.

To be able to model distance and represent events in a way that highlights the interaction in close vicinity to the

\footnotetext{
${ }^{4}$ The human is overtaking the robot while both are trying to reach the same goal.
}

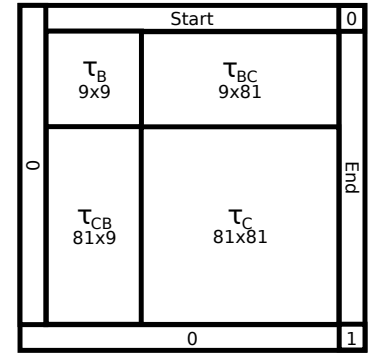

Fig. 4: The HMM transition matrix $\tau_{I}$ for $\mathrm{QTC}_{B C}$.

human we propose a probabilistic representation of QTC $\mathrm{C}_{B C}$. Compared to our previous work, we now model the proposed QTC $_{B C}$ instead of just QTC $_{C}$ which allows to dynamically switch between the two combined variants. This results in an extended transition probability matrix for $\tau_{I}$ (see Fig. 4).

Similar to the HMM based representation described in [10] we have initially modelled the "correct" emissions, e.g. $(+-)$ actually emits (+-), to occur with $95 \%$ probability and allow the model to account for classification errors with $5 \%$. Our HMM contains $\left|\tau_{I}\right|+2 \cdot\left|S_{I}\right|=1858+2 \cdot 90=2038$ legal transitions stemming from $\mathrm{QTC}_{B C}$ and the transitions from and to the start and end state, respectively.

To represent different HRSI behaviours, the HMM needs to be trained from the actual observed data. For each different behaviour to be represented, a separate HMM is trained using Baum-Welch training [15] (Expectation Maximisation) to obtain the appropriate transition and emission probabilities for the respective behaviour. In the initial pre-training model, the transitions that are valid according to our $\mathrm{QTC}_{B C}$ definition are modelled as equally probable (uniform distribution). We allow for pseudo transitions with a probability of $P_{p t}=$ $1 e^{-10}$ to overcome the problem of a lack of sufficient amounts of training data and unobserved transitions therein. To create the training set we have to transform the recorded data to $\mathrm{QTC}_{C}$ state chains that include the euclidean distance between $k$ and $l$ and define a threshold $d_{s}$ at which we want to transition from $\mathrm{QTC}_{B}$ to $\mathrm{QTC}_{C}$ and vice-versa. If $d(k, l)>d_{s}$, the values for $(c d)$ of the $\mathrm{QTC}_{C}$ representation are simply omitted and the remaining $(a b)$ 2-tuple will be represented by the $\mathrm{QTC}_{B}$ part of the transition matrix. If the distance crosses the threshold, it will be represented by one of the $\tau_{B C}$ or $\tau_{C B}$ transitions. QTC $C$ is used in the remainder of the cases. Afterwards, all distance values are removed from the representation because the QTC state chain now implicitly models $d_{s}$.

\section{EXPERIMENT}

To evaluate our QTC $_{B C}$ model we used the data of a previously conducted pilot study (initially described in [10]), investigating the movements of a human and a robot in a confined, shared space. The original aim of the study was to find hesitation signals in HRSI [16].

\section{A. Experiment Design}

In this study the participants where put into a hypothetical restaurant scenario together with a human-size robot (see 
Fig. 1). The experiment was situated in a large motion capture lab surrounded by 12 motion capture cameras, tracking the $x, y, z$ coordinates of human and robot with a rate of $50 \mathrm{~Hz}$. The physical set-up itself was comprised of two large boxes (resembling tables) and a bar stool (resembling a kitchen counter). The tables and the kitchen counter were on different sides of the room and connected via a $\sim 2.7 \mathrm{~m}$ long artificial corridor to elicit close encounters between the two agents while still being able to reliably track their positions (see Fig. 1). For this pilot study we had 14 participants (10 male, 4 female) who interacted with the robot for 6 minutes each. All of the participants were employees or students at the university and 9 of them have a computer science background; out of these 9 participants only 2 had worked with robots before. The robot and human were fitted with motion capture markers to track their $x, y$ coordinates for the QTC representation.

The robot was programmed to move autonomously back and forth between the two sides of the artificial corridor (kitchen and tables) using a state-of-the-art planner [17], [18]. Two different behaviours were implemented, i.e. adaptive and non-adaptive velocity control which were switched at random $(p=0.5)$ upon the robots arrival at the kitchen. The adaptive velocity control gradually slowed down the robot until it came to a complete stand still before entering the personal space [7] of the participant. The non-adaptive velocity control only regarded the human as a static obstacle trying to be as efficient as possible concerning the actual path planning. We chose to use these two distinct behaviours because they mainly differ in the speed of the robot and the distance it keeps to the human. Hence, they produce very similar, almost straight trajectories which allowed us to investigate the effect of distance and speed on the interaction while the participant was still able to reliably infer the robot's goal. This was necessary to find hesitation signals [16].

Before the actual interaction the human participant was told to play the role of a waiter together with a robotic coworker. This scenario allowed to create a natural form of pass-by interaction (see Fig. 1) between human and robot by sending the participants from the kitchen counter to the tables and back to deliver drinks while at the same time the robot was behaving in the described way. This task only occasionally resulted in encounters between human and robot but due to the incidental nature of these encounters and the fact that the participants were trying to reach their goal as efficient as possible we hoped to achieve a more natural and instantaneous participant reaction. All these specific behaviours are of no real importance for the qualitative representation because all participants showed very similar behaviour when circumventing the robot [10] and are just mentioned for the sake of completeness.

\section{B. Evaluation}

For the evaluation we followed a similar approach as described in [10]. We defined two virtual cut-off lines on either side of the corridor because we want to investigate close encounters between human and robot and therefore use only

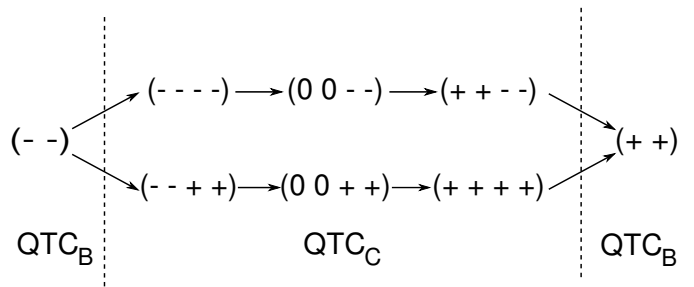

Fig. 5: Temporal sequence of $\mathrm{QTC}_{B C}$ for a head-on encounter. From left to right: approach, pass-by on the left or right side, moving away. Dashed lines represent instants where the distance threshold $d_{s}$ is crossed.

trajectories inside the corridor. Out of these trajectories we manually selected 71 head-on and 87 overtaking encounters and employed two forms of noise reduction on the recorded data. The actual trajectories were smoothed by averaging over the $x, y$ coordinates for $0.1 s, 0.2 s$, and $0.3 s$. The $z$ coordinate is not represented in QTC. To determine 0 QTC states - one or both agents move along $\vec{k} \vec{l}$ or along the two perpendicular lines (see Fig. 2) - we used three different quantisation thresholds: $1 \mathrm{~cm}, 5 \mathrm{~cm}$, and $10 \mathrm{~cm}$, respectively. Only if the movement of one or both of the agents exceeded these thresholds it was interpreted as a - or + QTC state. This smoothing and thresholding is necessary when dealing with discrete sensor data which otherwise would most likely never produce 0 states due to sensor noise.

To find appropriate distance thresholds for $\mathrm{QTC}_{B C}$ we evaluated distances for $0.1 m \leq d_{s} \leq 3 \mathrm{~m}$. The $d_{s}=$ $0.1 m$ threshold represents pure $\mathrm{QTC}_{B}$ because the robot and human are represented by their centre points, therefore, it is impossible for them to get closer than $10 \mathrm{~cm}$. On the other hand, the $d_{s}=3 m$ threshold represents pure QTC $_{C}$ because the corridor was only $\sim 2.7 \mathrm{~m}$ long.

To evaluate the generalisability and the meaningfulness of the representation, we used our previously described HMM based QTC ${ }_{B C}$ representation as a classifier to find similar encounters in our dataset. In order to show that this is possible, we employed $k$-fold cross validation with $k=5$, resulting in five iterations with a test set size of $20 \%$ of the selected trajectories. This was repeated ten times - to compensate for possible classification artefacts due to the random nature of the test set generation - resulting in 50 iterations over the selected trajectories. Subsequently, a normal distribution was fitted over the classification results to generate the mean and $95 \%$ confidence interval. This validation procedure was repeated for all nine smoothing and thresholding combinations.

\section{RESUlts \& Discussion}

To verify the effectiveness of $\mathrm{QTC}_{B C}$ with our HMM based approach we evaluated the classification rate for our two different classes of encounters, i.e. head-on and overtake, like in our previous work [10].

In order to show the benefits of $\mathrm{QTC}_{B C}$ we also evaluated passing on the left vs. passing on the right and adaptive vs. non-adaptive behaviour for the head-on cases. Fig. 5 shows 


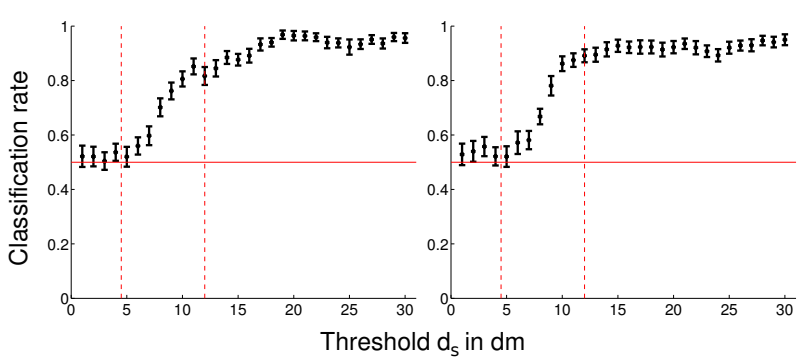

(a) Classification results for head-on passing on the left vs. right, lowest and highest smoothing parameters. Left $1 \mathrm{~cm}$ and $0.1 \mathrm{~s}$ smoothing, right $10 \mathrm{~cm}$ and $0.3 \mathrm{~s}$ smoothing.

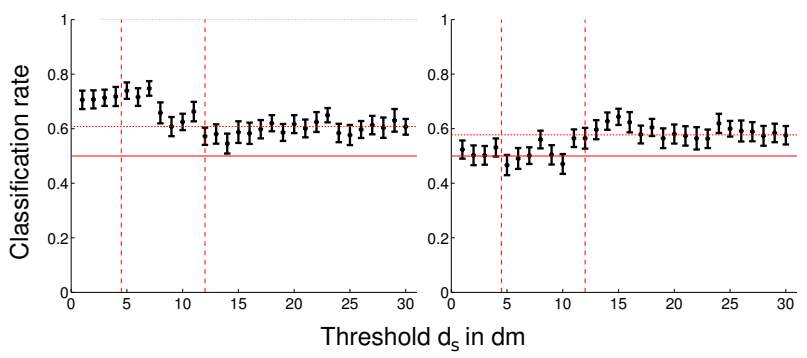

(b) Classification results for head-on adaptive vs. non-adaptive. Left: $5 \mathrm{~cm}$ and $0.2 \mathrm{~s}$ smoothing, right: $1 \mathrm{~cm}$ and $0.3 \mathrm{~s}$ smoothing. Horizontal dotted line: Classification result from [10]

Fig. 6: Classification results. The point represents the mean and the errobar the $95 \%$ confidence interval. Horizontal line: Null Hypothesis. Vertical dashed lines: Hall's intimate $(45 \mathrm{~cm})$ and personal $(1.2 \mathrm{~m})$ space [7].

an example of a resulting $\mathrm{QTC}_{B C}$ representation of a headon encounter.

\section{A. Results}

Table Ia shows the minimum and maximum classification rates $(\mu)$ for the general head-on vs. overtaking case and the respective $\mathrm{QTC}_{B C}$ thresholds $\left(d_{s}\right)$. For the majority of the different smoothing levels $(7 / 9)$, the best classification results were achieved using distance thresholds of $0.1 \mathrm{~m} \leq$ $d_{s} \leq 0.6 m$.

The comparison of passing on the left vs. passing on the right, is shown in Table Ib. All of the results show bad performance if $d_{s} \leq 0.7 \mathrm{~m}$, and high classification results for values of $d_{s} \geq 0.9 \mathrm{~m}$. Fig. 6a shows two typical results. The left hand side shows the classification rates for the lowest smoothing settings and the right hand side shows the results for the highest smoothing level. In all of the cases a sudden increase in performance - jumping from $\mu \approx 0.5$ to $\mu>0.8$ - can be seen at $0.9 m \leq d_{s} \leq 1.2 m$.

The third case, adaptive vs. non-adaptive robot behaviour in head-on encounters, is shown in Table Ic. The best results were achieved at distances of $0.1 m \leq d_{s} \leq 0.7 \mathrm{~m}$, all but one lying on the diagonal of Table Ic. Fig. $6 b$ shows two exemplary results. The left hand side depicts the best classification result with classification rates of up to $\mu=0.748$ for $d_{s}=0.7 \mathrm{~m}$. The right hand side shows the results for a smoothing level that did not yield the best results for low but medium distance threshold of $d_{s}=1.5 \mathrm{~m}$ with a classification rate of $\mu=0.643$.

\section{B. Discussion}

Our presented approach $\mathrm{QTC}_{B C}$ uses $d(k, l)_{t-1}$ and $d(k, l)_{t}$ to determine if the representation should transition from $\mathrm{QTC}_{B}$ to $\mathrm{QTC}_{C}$ or vice-versa. This might lead to unwanted behaviour if the distance $d(k, l)$ oscillates around $d_{s}$. Due to the manual selection of data, we did not face such problems in this evaluation but it is a clear limitation of this approach which has to be overcome for "live" applications. For the following discussion we can assume that this had no negative effect on the presented data.

The classification of head-on vs. overtaking produced similar results to our previous evaluation [10]. This shows that QTC $_{B C}$ does not decrease the generalisability of our HMM based representation for this two class example. We have also seen that there are cases where pure $\mathrm{QTC}_{B}$ outperforms pure $\mathrm{QTC}_{C}$. This is not surprising because the main difference of overtaking and head-on lies in the $(a b) 2$ tuple of $\mathrm{QTC}_{B}$, i.e. both agents move in the same direction, e.g. $(-+)$, vs. both agents are approaching each other $(--)$. The $(c d)$ QTC $_{C}$ information can therefore be disregarded in most of the cases. This indicates that $\mathrm{QTC}_{B}$ would be sufficient to classify head-on and overtaking scenarios but would of course not contain enough information to generate an appropriate behaviour. QTC $\mathrm{TC}_{B C}$ allows to incorporate the information about which side robot and human should use to pass each other and the distance at which to start circumventing. Since all of the found classification results were significantly different from $p=0.5$ - the Null Hypothesis $\left(H_{0}\right)$ for a two class problem - this distance can be chosen to represent a meaningful value like Hall's personal space.

The comparison of left vs. right pass-by actions in headon encounters shows that using pure $\mathrm{QTC}_{B}$ does, not surprisingly, yield bad results because the most important information - on which side the robot an the human pass by each other - is completely omitted. All the classification results show that more information about the values of $(c d)$ increases the performance of the classification. On the other hand, the results also show that the largest increase in performance of the classifier happens at a distance of $0.9 m \leq d_{s} \leq 1.2 m$ (see Fig. 6a), which resembles Hall's personal space of $1.2 \mathrm{~m}$ [7]. These results show that the human interaction partner granted the robot its personal space or tried to avoid having the robot violate their own. Judging from our data, the results indicate that information about the $(c d)$ 2-tuple is most important if both agents enter, or are about to enter, each others personal spaces. The information before and after this threshold can be disregarded and is not important for the reliable classification of these two behaviours.

Using the previous probabilistic model of $\mathrm{QTC}_{C}$, it was not possible to reliably distinguish between the two behaviours the robot showed during the experiment [10]. We investigated if $\mathrm{QTC}_{B C}$ would sufficiently highlight the difference between these two classes to enable a correct classification. Indeed, the results indicate that using a very low distance threshold $d_{s}$ enables QTC $_{B C}$ to distinguish 
TABLE I: Classification results

(a) Head-on vs. Overtake

\begin{tabular}{|c|c|c|c|c|c|c|c|}
\hline \multicolumn{2}{|c|}{ Smoothing } & \multicolumn{2}{c|}{$0.1 s$} & \multicolumn{2}{c|}{$0.2 s$} & \multicolumn{2}{c|}{$0.3 s$} \\
\cline { 2 - 8 } & Res. & $\mu$ & $d_{s}$ & $\mu$ & $d_{s}$ & $\mu$ & $d_{s}$ \\
\hline \multirow{2}{*}{$1 \mathrm{~cm}$} & $\min$ & 0.90 & 0.7 & 0.89 & 1.0 & 0.91 & 0.7 \\
\cline { 2 - 8 } & $\max$ & 0.97 & 3.0 & 0.96 & $\mathbf{0 . 6}$ & $\mathbf{0 . 9 8}$ & $\mathbf{2 . 2}$ \\
\hline \multirow{2}{*}{$5 \mathrm{~cm}$} & $\min$ & 0.84 & 0.8 & 0.88 & 0.8 & 0.87 & 0.7 \\
\cline { 2 - 8 } & $\max$ & 0.92 & $\mathbf{0 . 5}$ & 0.97 & $\mathbf{0 . 1}$ & 0.94 & $\mathbf{0 . 1}$ \\
\hline \multirow{2}{*}{$10 \mathrm{~cm}$} & $\min$ & 0.70 & 2.0 & 0.79 & 1.2 & 0.79 & 0.9 \\
\cline { 2 - 8 } & $\max$ & 0.82 & $\mathbf{0 . 3}$ & 0.87 & $\mathbf{0 . 5}$ & 0.89 & $\mathbf{0 . 4}$ \\
\hline
\end{tabular}

(b) Head-on: Left vs. Right

\begin{tabular}{|c|c|c|c|c|c|}
\hline \multicolumn{2}{|c|}{$0.1 s$} & \multicolumn{2}{c|}{$0.2 s$} & \multicolumn{2}{c|}{$0.3 s$} \\
\hline$\mu$ & $d_{s}$ & $\mu$ & $d_{s}$ & $\mu$ & $d_{s}$ \\
\hline $\mathbf{0 . 5 0}$ & $\mathbf{0 . 3}$ & 0.58 & 0.3 & 0.52 & 0.2 \\
\hline $\mathbf{0 . 9 7}$ & $\mathbf{1 . 9}$ & 0.95 & 2.4 & 0.96 & 2.3 \\
\hline 0.41 & 0.2 & 0.41 & 0.2 & 0.49 & 0.2 \\
\hline 0.90 & 2.9 & 0.93 & 2.8 & 0.94 & 2.9 \\
\hline 0.50 & 0.2 & 0.43 & 0.1 & $\mathbf{0 . 5 2}$ & $\mathbf{0 . 5}$ \\
\hline 0.92 & 3.0 & 0.90 & 1.2 & $\mathbf{0 . 9 5}$ & $\mathbf{3 . 0}$ \\
\hline
\end{tabular}

(c) Head-on: Adaptive vs. Non-Adaptive

\begin{tabular}{|c|c|c|c|c|c|}
\hline \multicolumn{2}{|c|}{$0.1 s$} & \multicolumn{2}{c|}{$0.2 s$} & \multicolumn{2}{c|}{$0.3 s$} \\
\hline$\mu$ & $d_{s}$ & $\mu$ & $d_{s}$ & $\mu$ & $d_{s}$ \\
\hline $\mathbf{0 . 4 6}$ & $\mathbf{1 . 4}$ & 0.48 & 1.8 & 0.47 & 0.5 \\
\hline $\mathbf{0 . 6 6}$ & $\mathbf{0 . 1}$ & 0.60 & 0.8 & 0.64 & 1.5 \\
\hline $\mathbf{0 . 5 2}$ & $\mathbf{1 . 0}$ & $\mathbf{0 . 5 5}$ & $\mathbf{1 . 4}$ & 0.54 & 1.3 \\
\hline $\mathbf{0 . 6 9}$ & $\mathbf{1 . 5}$ & $\mathbf{0 . 7 5}$ & $\mathbf{0 . 7}$ & 0.72 & 0.5 \\
\hline 0.46 & 1.2 & 0.49 & 0.8 & $\mathbf{0 . 5 9}$ & $\mathbf{1 . 6}$ \\
\hline 0.60 & 1.8 & 0.64 & 1.0 & $\mathbf{0 . 7 4}$ & $\mathbf{0 . 7}$ \\
\hline
\end{tabular}

between these two cases for some of the smoothing levels. In Fig. $6 \mathrm{~b}$ you can see the results from our previous work [10] visualised by a horizontal dotted line and that some of the results are significantly different from the previous ones. Like for head-on vs. overtake, the main difference between the adaptive and non-adaptive behaviour lies in the (a b) 2-tuple, i.e. (--) vs. $(-0)$, but, in contrast to that, the classification rate for adaptive vs. non-adaptive drops to $p \approx 0.5\left(H_{0}\right)$ at $d_{s}=1.3 \mathrm{~m}$. On the other hand, there is also an interesting example where this does not hold true and we see a slight increase in classification rate at $d_{s}=1.5 \mathrm{~m}$ which was the stopping distance of the robot. The results for adaptive vs. non-adaptive also seem to be very dependent on the smoothing parameters (see Table Ic) and are therefore still quite inconclusive.

\section{CONClusion \& Future Work}

We presented a novel approach for implicitly modelling social distances in QTC by combining different variants of the calculus, i.e. $\mathrm{QTC}_{B}$ and $\mathrm{QTC}_{C}$, into one integrated QTC $_{B C}$ model. This incorporation of the distance is a first step to employ learned representations of HRSI for the generation of appropriate robot behaviour. To further improve this representation, we will work on a generalised version of our presented QTC $_{B C}$ to deal with different and possibly multiple variants of QTC, which are not restricted to $\mathrm{QTC}_{B}$ and $\mathrm{QTC}_{C}$, based also on other metrics beside Hall's social distances to allow behaviour analysis according to multiple HRSI measures.

The resulting HMM based probabilistic model of $\mathrm{QTC}_{B C}$, using a distance threshold $d_{s}=1.2 \mathrm{~m}$ (Hall's personal space), is able to create a compact qualitative representation of HRSI only representing the essence of pass-by situations by filtering unwanted information. Our experiments showed that this representation is able to classify two of the three presented two-class problems correctly. The results for the third classification problem, i.e. adaptive vs. non-adaptive, showed improvements compared to previous work. HMM based $\mathrm{QTC}_{B C}$ is therefore able to create a representation that is as compact as possible and yet sufficiently complex to still reliably classify the different encounters.

A subsequent user study will show if our model is also able and suited to generate behaviour for a mobile robot.

\section{REFERENCES}

[1] J. Borenstein and Y. Koren, "Real-time obstacle avoidance for fast mobile robots," IEEE Transactions on Systems, Man and Cybernetics, vol. 19 , no. 5, pp. 1179-1187, 1989.

[2] R. Simmons, "The curvature-velocity method for local obstacle avoidance," in IEEE International Conference on Robotics and Automation, vol. 4, no. April. Minneapolis, MN: IEEE, 1996, pp. 3375-3382.

[3] E. Sisbot, L. Marin-Urias, R. Alami, and T. Simeon, "A Human Aware Mobile Robot Motion Planner," IEEE Transactions on Robotics, vol. 23, no. 5, pp. 874-883, Oct. 2007.

[4] M. Yoda and Y. Shiota, "Analysis of human avoidance motion for application to robot," in Proceedings 5th IEEE International Workshop on Robot and Human Communication. RO-MAN'96 TSUKUBA. IEEE, 1996, Conference proceedings (whole), pp. 65-70.

[5] D. J. Feil-Seifer and M. J. Matarić, "People-Aware Navigation For Goal-Oriented Behavior Involving a Human Partner," in Proceedings of the Int. Conf. on Development and Learning, Frankfurt a.M., Germany, Aug. 2011.

[6] T. Ducourant, S. Vieilledent, Y. Kerlirzin, and A. Berthoz, "Timing and distance characteristics of interpersonal coordination during locomotion," Neuroscience Letters, vol. 389, no. 1, pp. 6-11, Nov. 2005.

[7] E. T. Hall, The hidden dimension. Anchor Books New York, 1969.

[8] M. Hanheide, A. Peters, and N. Bellotto, "Analysis of human-robot spatial behaviour applying a qualitative trajectory calculus," in $R O$ MAN, 2012 IEEE. IEEE, 2012, pp. 689-694.

[9] N. Bellotto, M. Hanheide, and N. Van de Weghe, "Qualitative design and implementation of human-robot spatial interactions," in Proc. of Int. Conf. on Social Robotics (ICSR), 2013.

[10] C. Dondrup, N. Bellotto, and M. Hanheide, "A probabilistic model of human-robot spatial interaction using a qualitative trajectory calculus," in 2014 AAAI Spring Symposium Series, 2014.

[11] N. Van de Weghe, "Representing and reasoning about moving objects: A qualitative approach," Ph.D. dissertation, Ghent University, 2004.

[12] E. Pacchierotti, H. I. Christensen, and P. Jensfelt, "Evaluation of passing distance for social robots," in The 15th IEEE International Symposium on Robot and Human Interactive Communication, ROMAN 2006, 2006, pp. 315-320.

[13] C. Lichtenthäler, A. Peters, S. Griffiths, and A. Kirsch, "Social navigation-identifying robot navigation patterns in a path crossing scenario," ICSR. Springer, 2013.

[14] A. G. Cohn and J. Renz, "Chapter 13 Qualitative Spatial Representation and Reasoning," in Handbook of Knowledge Representation, F. van Harmelen, V. Lifschitz, and B. Porter, Eds. Elsevier, 2008, vol. 3, pp. 551-596.

[15] G. A. Fink, Markov Models for Pattern Recognition. Springer-Verlag Berlin Heidelberg, 2008.

[16] C. Dondrup, C. Lichtenthäler, and M. Hanheide, "Hesitation signals in human-robot head-on encounters: a pilot study," in Proceedings of the 2014 ACM/IEEE international conference on Human-robot interaction. ACM, 2014, pp. 154-155.

[17] D. Fox, W. Burgard, and S. Thrun, "The dynamic window approach to collision avoidance," IEEE Robotics \& Automation Magazine, vol. 4, no. 1, pp. 23-33, 1997.

[18] S. Thrun, W. Burgard, and D. Fox, Probabilistic robotics. MIT press, 2005. 